\title{
Hereditary thrombophilia and adverse pregnancy outcomes
}

\author{
Valentin Friptu, Diana Mitriuc, Olga Popusoi \\ Department of Gynecology, Obstetrics and Human Reproduction \\ Nicolae Testemitanu State University of Medicine and Pharmacy, Chisinau, the Republic of Moldova \\ Authors' ORCID iDs, academic degrees and contributions are available at the end of the article \\ *Corresponding author - Diana Mitriuc, e-mail: diana.mitriuc@gmail.com \\ Manuscript received April 06, 2021; revised manuscript July 05, 2021; published online September 10, 2021
}

\begin{abstract}
Background: Multiple studies have found a relatively increased risk of placenta-mediated pregnancy complications in women with congenital thrombophilia, especially early recurrent pregnancy loss, fetal loss, early-onset preeclampsia, intrauterine growth restriction, and premature abruption of normally positioned placenta. However, the extent of the association and the absolute risk are very modest, but they significantly increase in pregnant women with severe obstetric complications.

Conclusions: There is convincing evidence that deficiency of natural anticoagulants (antithrombin, protein C, protein S) is a risk factor for late fetal loss. Factor V Leiden G1691A gene mutation and prothrombin G20210A gene mutation are associated with a double risk for early and unexplained recurrent pregnancy loss and for non-recurrent late fetal loss. The association of congenital thrombophilia with preeclampsia is much more uncertain, being probably limited factor V Leiden G1691A gene mutation and more severe cases of preeclampsia. Fewer data are available on intrauterine growth restriction (IUGR) and premature abruption of the normally positioned placenta. There is insufficient evidence to suggest an association of other forms of congenital thrombophilia with adverse pregnancy outcomes. In addition, genetic and epidemiological research suggests that placenta-mediated pregnancy complications are of polygenic multifactorial etiology, with a risk determined by the interaction of multiple genetic variants and other risk factors.

Key words: pregnancy complications, hereditary thrombophilia, recurrent pregnancy loss.
\end{abstract}

Cite this article

Friptu V, Mitriuc D, Popusoi O. Hereditary thrombophilia and adverse pregnancy outcomes. Mold Med J. 2021;64(3):68-77. https://doi.org/10.52418/ moldovan-med-j.64-3.21.13.

\section{Introduction}

Congenital thrombophilia, in general agreement with the literature, in addition to its established role in the pathogenesis of venous thromboembolism (VTE) increases the risk of gestational vascular complications and adverse pregnancy outcomes. The role of congenital thrombophilia in gestational vascular disorders and adverse pregnancy outcomes (miscarriage, fetal loss, preeclampsia, intrauterine growth restriction and premature abruption of the normally positioned placenta) has been evaluated in several studies and the results are inhomogeneous and contradictory [1-3]. The evaluation of a large number of studies in the literature on congenital thrombophilia in pregnancy does not assign an overall incidence of risk, indicating only an absolutely low risk in pregnant women and in the fetus during pregnancy. It is still difficult to establish the exact quantitative data of the risk for certain adverse events in the presence of these mutations $[4,5]$. In addition, the size of estimated risks varies, due to the heterogeneity of different study designs [6].

Major forms of hereditary thrombophilia, currently recognized as independent risk factors for VTE, include procoagulant abnormalities - factor V Leiden G1691A (FVL) gene mutation of homozygous or heterozygous type, homo- zygous or heterozygous prothrombin G20210A gene mutation, endogenous deficiency of natural anticoagulants - antithrombin (AT), protein $\mathrm{C}$ and protein $\mathrm{S}$, and mutation of the methylenetetrahydrofolate reductase (MTHFR) C677T gene. The most common forms of congenital thrombophilia are the FVL G1691A gene mutation and the prothrombin G20210A gene mutation of the heterozygous type, the other types of congenital thrombophilia (AT deficiency, protein C and protein $\mathrm{S}$ deficiency) have a higher thrombogenic potential, but are less common [ 4, 7-10].

The data below summarize the prevalence of the most important types of congenital thrombophilia, including recently detected, a brief review of the available evidence for each congenital thrombophilia in relation to adverse pregnancy outcomes and the risk of VTE.

FVL G1691A gene mutation. The risk of developing VTE increases 2-7 times in people with heterozygous FVL G1691A gene mutation and 40-80 times in people with homozygous FVL G1691A gene mutation [7]. About $40-44 \%$ of women with VTE, in pregnancy or postpartum, are carriers of the FVL G1691A gene mutation and most are heterozygous $[4,11]$.

Data on the relationship between the FVL G1691A gene mutation and fetal loss are inconsistent. In general, the FVL 
G1691A gene mutation contributes to a small increase in the risk of miscarriage and recurrent pregnancy loss (RPL) [1, $7,12,13]$. In terms of other parameters of obstetric morbidity, a recent meta-analysis suggests that there is a significant increase in the frequency of obstetric complications in general, prenatal mortality (stillbirth), severe preeclampsia, intrauterine growth restriction (IUGR), newborns small for gestational age and premature abruption of the normally positioned placenta (PANPP) in carriers of the FVL G1691A gene mutation $[7,12]$.

Two recent and comprehensive literature reviews, which included prospective, retrospective case-control and cohort studies with a moderate heterogeneity, established that carriers of the FVL G1691A gene mutation had a relatively high risk of RPL (1.52- 2.02 times higher). However, the absolute risk of pregnancy loss in women with the FVL G1691A gene mutation is low (4.2\%) [14].

There is no significant association between the FVL G1691A gene mutation and preeclampsia, between the FVL G1691A gene mutation and IUGR or newborns small for gestational age [14]. The association between PANPP and the FVL G1691A gene mutation is also insufficient [8].

However, the association with severe preeclampsia of the FVL G1691A gene mutation was assessed in a meta-analysis of case-control studies and found a strong association; the Odds Ratio (OR) ranges from 2.04 to 3.0 [13]. A systematic literature review and a recent meta-analysis, estimating 42 case-control and cohort studies, published in 2016, found a significant association of the FVL G1691A gene mutation with newborns small for gestational age (OR 1.40) and the lack of association of the FVL G1691A gene mutation with premature birth [15].

Prothrombin G20210A gene mutation. The risk of VTE in carriers of prothrombin G20210A gene mutation is 2-5 times higher, and in combination with the FVL G1691A gene mutation - 20 times higher [7]. The heterozygous type of prothrombin G20210A gene mutation has a 3-8-fold higher risk, and the homozygous type - 18-80-fold higher risk of thromboembolic events [9].

A systematic literature review found an OR of 2.49 for early pregnancy loss, 2.7 for early RPL, 2.66 for late pregnancy loss, 2.54 for preeclampsia, 2.92 in newborns small for gestational age and 7.71 for PANPP in pregnant women with the prothrombin G20210A gene mutation, compared to pregnant women who do not have this form of congenital thrombophilia [13].

Data cumulated from several studies indicate a significant association between the prothrombin G20210A gene mutation and RPL, total and severe preeclampsia. Although the relationship between the prothrombin G20210A gene mutation and VTE during pregnancy is clear, the results of some studies do not confirm the impact of this mutation on obstetric complications: early RPL, preeclampsia, including early or severe onset preeclampsia, PANPP, IUGR, and newborns small for gestational age $[1,7,8,12,14]$.

Protein $\mathrm{C}$ deficiency. In general, studies have not found a clear association between protein $\mathrm{C}$ deficiency and RPL
$[1,4,7]$, but in the case of the association of protein $S$ and protein $\mathrm{C}$ deficiency, there is a correlation with an increased incidence of stillbirth [4]. There are no studies that have found an association between protein $\mathrm{C}$ deficiency and early pregnancy loss, IUGR or PANPP. Protein C deficiency has been associated with an increased risk of miscarriage in the second trimester, preeclampsia, and stillbirth [8].

Protein S deficiency. A literature review, published in 2002, evaluating 3-5 relevant studies, found an increased risk of preeclampsia (12.7 times higher) with an absolute risk of $12.3 \%$ and an increase in stillbirths (16.2 times higher) with an absolute risk of $6 \%$ in pregnant women with protein $S$ deficiency. There are no studies that have found an association between protein S deficiency and early pregnancy loss, IUGR or PANPP [8].

Given the low prevalence and limited data, definitive conclusions on the effect of protein $\mathrm{C}$ and protein $\mathrm{S}$ on RPL and other pregnancy complications cannot be reached and further investigations are needed $[1,3]$.

AT deficiency was the first hereditary thrombophilia identified, which significantly increases the risk of VTE. Being the most thrombogenic, it has the highest risk of VTE among hereditary thrombophilias and often requires longterm anticoagulant therapy. The lifetime VTE risk accounts for $70-90 \%$, being particularly high in pregnancy, postpartum period and after major surgery [7].

Data on the role of AT deficiency in fetal loss (RPL, late loss) are contradictory. Some studies have found that women with AT-III deficiency have an increased risk of embryonic death and fetal death compared to the general population [7, 8]. The results obtained in other studies suggest an association between AT deficiency and pregnancy loss, but the definitive causal relationship is not yet established. AT-III deficiency is rarely associated with severe preeclampsia, IUGR or PANPP, but this could be «false negative» due to the low prevalence of this form of thrombophilia [7].

MTHFR C677T gene mutation. Hyperhomocysteinemia is an independent risk factor for VTE. Although several studies have not found a firm relationship between the homozygous MTHFR C677T gene mutation or elevated homocysteine levels with adverse pregnancy outcomes [1, 7], a recent meta-analysis revealed an association of the MTHFR C677T gene mutation with severe preeclampsia [12].

Another meta-analysis, which included 26 case-control studies with 2120 cases and 2949 controls (1997-2005), and some recent studies found that the MTHFR C677T gene mutation is a genetic risk factor of unexplained RPL (two or more consecutive miscarriages) only in the Chinese and Iranian population, but not in the population of European countries $[16,17]$.

Two recent meta-analyses, which included 27 and 37 studies (1997-2011 and 1997-2012) with 2427 and 3559 cases, 2120 and 5097 controls (published in 2012 and 2013), identified a significant association between the MTHFR C677T gene mutation and unexplained RPL (two or three and more consecutive pregnancy losses) in the East Asian subgroup (Chinese, Japanese and Korean population) and in 
the mixed subgroup (Indian, Brazilian, Bahraini, Mexican and Egyptian population), but not in the Caucasian population, including the population of European origin. In addition, the study found a genetic heterogeneity between the ethnic groups $[18,19]$.

Three recent meta-analyses, published in 2014 and 2015, which included a substantial number of cases and controls, revealed statistically significant results of the relationship between the MTHFR C677T gene mutation and hypertension in the population, hypertension in pregnancy and preeclampsia, in particular among Asians and Caucasians. The authors consider that this polymorphism is an independent risk factor for hypertension and preeclampsia [20,21].

The results of the Hordaland Homocysteine Study, conducted among 5883 women aged 40-42, with 14492 pregnancies, and published in 2004, revealed that maternal polymorphism MTHFR C677T is associated with increased pregnancy complications in general, increased risk of PANPP and, possibly with IUGR or newborns small for gestational age [22].

Therefore, all types of congenital thrombophilia are associated with an increased risk of VTE during pregnancy. Several studies confirmed the increased risk of obstetric complications in pregnant women with congenital thrombophilia, but despite the increased relative risk, the absolute risk of VTE and negative pregnancy outcomes is reduced. Moreover, the risk estimation largely depends on the type of hereditary thrombophilia analyzed, the methodological criteria applied for patient selection, the ethnic groups included in the study, the differences in sensitivity and specificity of laboratory methods of thrombophilia detection and the polygenic multifactorial etiology of pregnancy complications.

There is convincing evidence that deficiency of natural anticoagulants (AT, protein C, protein S) is a risk factor for late fetal loss. The FVL G1691A gene mutation and the prothrombin G20210A gene mutation are associated with a double risk for unexplained early RPL and non-recurrent late fetal loss. The association of congenital thrombophilia with preeclampsia is much more uncertain, being probably limited to the mutation of the FVL G1691A gene and more severe cases of preeclampsia. Fewer data are available about IUGR and PANPP. The absolute risk of VTE and negative pregnancy outcomes increase significantly in case of a combination of two or more forms of hereditary thrombophilia.

Contemporary technologies have recently contributed to the discovery of new forms of hereditary thrombophilia: factor V A4070G gene mutation, factor XIII G103T gene mutation, MTHFR A1298C gene mutation, methionine synthase (MTR) A2756G gene mutation, methionine synthase reductase (MTR) A66G gene mutation, plasminogen activator inhibitor (PAI-1) gene mutation, cystathionine betasynthetase (CBS) 844ins68pb gene mutation. However, information on these polymorphisms is limited, and their role in the development of unexplained RPL and other obstetric complications is still controversial $[23,24]$.

The factor V A4070G gene mutation has a pathological role, causing a moderate resistance to activated protein $\mathrm{C}$ and interacting with the FVL G1691A gene mutation to produce a more resistant phenotype to activated protein C. As no significant increase in the prevalence was identified, the presence of the factor V A4070G gene mutation is unlikely to be an independent risk factor for pregnancy-associated complications. It is controversial whether this mutation is associated with an increased thrombotic risk in the absence of the FVL G1691A gene mutation [9, 25].

The heterozygous MTHFR A1298C gene mutation has a strong association and is a genetic risk factor for RPL [17, 26] and PANPP [27]. The association of RPL with MTHFR A1298C polymorphism is found in previously published studies and in a meta-analysis of 5 studies, published in 2013 $[26,28]$.

However, recent meta-analyses and case-control studies did not identify any association between the MTHFR A1298C gene mutation and unexplained RPL (two or three and more consecutive pregnancy losses) $[18,29,30]$, preeclampsia [20], IUGR or newborns small for gestational age [22]. In contrast, this mutation may have a protective effect by raising serum folic acid levels and decreasing homocysteine levels in the serum $[31,32]$, and may significantly reduce the risk of pregnancy complications in asymptomatic nulliparous pregnant women associated with MTHFR C677T polymorphism [33], although these results require confirmation.

The CBS 844ins68 gene mutation is not a genetic risk factor for venous thrombosis [34], although the results of some studies suggest possible effects of this polymorphism on the risk of idiopathic miscarriage, but it does not have a significant certainty [35].

MTR A2756G gene mutation. The role of the MTR A2756G gene polymorphism in the development of hyperhomocysteinemia is still controversial. Some studies did not find any significant risk of hyperhomocysteinemia in people with the MTR A2756G gene mutation. The effect of reducing homocysteine levels is synergistic in people with both mutations (CBS 844ins68 gene mutation and MTR A2756G gene mutation), and homocysteine levels are much lower than in people with one of these polymorphisms [36]. Other studies have shown that the MTR A2756G polymorphism independently increases the risk of hyperhomocysteinemia [31, 32].

The methionine synthase-reductase gene mutation (MTRR A66G) does not affect folic acid levels or the incidence of folate deficiency, has no effect on plasma concentration of total homocysteine, folate or vitamin $\mathrm{B}_{12}$ in pregnant women [37] or the Chinese population [31, 32]. However, some studies have shown that the MTRR A66G gene polymorphism and folic acid deficiency have been significantly associated with high serum levels of total homocysteine [38].

The interactions between gene polymorphisms regulating folic acid metabolism and homocysteine have significant interactive effects leading to a dramatic increase in the risk of folate deficiency with the rise of serum homocysteine concentration. Furthermore, patients with two or more risk genotypes (MTHFR C677T, MTHFR A1298C, MTR A2756G, MTRR A66G gene mutations) have higher folate deficiency rates, especially those with the combination of 
MTHFR C677T and MTRR A66G, between MTHFR C677T and MTHFR A1298C or all four risk genotypes [31, 32].

Glycoprotein IIb / IIIa mutation. Although information on the role of glycoprotein IIb / IIIa mutation in the development of pregnancy complications is limited, some studies have found a significant association of glycoprotein IIIa gene mutation with RPL [28], and PLA2 gene polymorphism is associated with miscarriage between the 10th and the 20th week of pregnancy [9].

$\boldsymbol{\beta}$-fibrinogen gene G455A mutation. Elevated fibrinogen levels are associated with an increased risk (4-fold higher) of developing VTE, possibly through increased blood viscosity and platelet aggregation $[7,9]$. The relationship between $\beta$-fibrinogen G-455A polymorphism and RPL is controversial. Some studies have found a significant association of $\beta$-fibrinogen gene G-455A mutation with RPL and may predict an increased risk of repeated pregnancy loss [28, 39]. However, a recent meta-analysis, published in 2015, did not reveal any statistically significant association between $\beta$-fibrinogen G-455A polymorphism and RPL, including after the separate analysis of the Asian and Caucasian population [40].

PAI-1 gene mutation. A recent, comprehensive up-todate meta-analysis, which examined a total of 3561 cases and 5693 controls, revealed a statistically significant correlation of PAI-1 4G/5G gene polymorphism with VTE in the general population. This association does not depend on ethnicity - the increased risk of VTE has been found in Asians and Caucasians, especially in people with the association of other genetic thrombophilic disorders [41].

A recent systematic analysis and meta-analysis, published in 2015, which included 18 case-control studies (conducted in 12 countries between 2003 and 2014) with a total of 3684 cases and 2208 controls, found a significant association between PAI-1 4G/5G polymorphism and RPL risk (two or more consecutive pregnancy losses in the first two trimesters). However, there was a statistically significant heterogeneity between the included studies [42].

Another recent meta-analysis, published in 2015, which included 22 case-control and cohort studies (2003-2014) with a total of 4306 cases and 3076 controls, also found a significant association between PAI-1 4G/5G polymorphism and RPL risk. The race-based analysis found that PAI-1 $4 \mathrm{G} / 5 \mathrm{G}$ polymorphism was significantly associated with an increased risk of RPL in Caucasians ( $\mathrm{OR}=2.23$; 95\% CI 1.44$3.46 ; \mathrm{P}=0.0003)$ and no significant association was identified in Asians $(\mathrm{OR}=1.47 ; 95 \% \mathrm{CI} 0.84-2.59 ; \mathrm{P}=0.18)$ [43].

However, several prospective case-control and cohort studies did not find any positive association of PAI-1 4G/5G polymorphism (isolated or in combination with other forms of congenital thrombophilia) with an increased risk of RPL and other adverse effects of pregnancy, although in most studies the small sample size limits the interpretation of these results [44-47].

Factor XIII V34L gene mutation. The association of V34L polymorphism of coagulation factor XIII with an increased risk of RPL is still debatable and controversial. According to the results of case-control studies, the factor XIII
V34L gene mutation of heterozygous type and, to a lesser extent, of homozygous type, is significantly associated with unexplained RPL and can be considered a risk factor for RPL, being associated with the early development of RPL [48]. A recent meta-analysis, published in 2015, also demonstrated a close association of the factor XIII V34L gene mutation with RPL [40].

However, some studies have not found any association of the factor XIII V34L mutation (isolated or in combination with other forms of congenital thrombophilia) with RPL. However, the small sample size and the inclusion in studies of different ethnic groups limited the interpretation of the results $[45,49]$.

Therefore, discrepancies in the relationship between different forms of hereditary thrombophilia and placentamediated pregnancy complications can be explained by the small sample size, the difference between the ethnic groups, and the geographical variation of different cohort studies. Large case-control, prospective, and population-based studies are needed to confirm or to disprove the association of more recently discovered hereditary thrombophilia with adverse pregnancy outcomes.

The success achieved in the last decades in the field of molecular medicine, biology and medical genetics has contributed to the evaluation from fundamentally new perspectives of the pathogenesis of many obstetric complications, which increase fetal, infant and maternal mortality. RPL or recurrent miscarriage, pregnancy stopped in evolution, premature birth, intrauterine fetal death, PANPP, IUGR, severe preeclampsia, eclampsia, thromboembolism are still very current problems for modern obstetrics. The discovery of different forms of acquired and hereditary thrombophilia elucidated much more widely the pathogenesis of multiple diseases, including in general obstetrics $[7,50]$.

Although the contribution of congenital thrombophilia to negative pregnancy outcomes is controversial, a number of complications (fetal loss, preeclampsia, PANPP, IUGR) have been associated with congenital thrombophilia [51]. The association between pregnancy complications and different forms of congenital thrombophilia, both quantitatively and qualitatively, is briefly described below.

Recurrent miscarriage. The term miscarriage is often used to define pregnancy loss from conception to 20 weeks of gestation. Subsequently, the loss of pregnancy is called the death of the fetus or stillbirth. Early pregnancy loss is defined as the lack of fetal cardiac activity up to 12 weeks of gestation, and late pregnancy loss - the lack of fetal cardiac activity after 12 weeks of gestation $[52,53]$.

Common definitions of RPL, also known as repeated miscarriage, include the following: 1) two or more miscarriages in the first trimester (up to 12 weeks of gestation), documented by ultrasound or histopathological examination, 2) three or more consecutive pregnancy losses up to 10-12 weeks of gestation, which are not necessarily intrauterine $[1,50,54]$.

The hemostatic system plays an important role in the normal development and success of pregnancy, the implantation process, the invasion and normal development of the 
trophoblast and placentation. The implantation of the fertilized egg in the decidua establishes a contact between the fetus, the placenta and the maternal circulation which is crucial for the pregnancy success. Prothrombotic changes and thrombosis can interfere with these processes, contributing to severe consequences, including miscarriage, and may account for many unexplained cases of previous RPL $[3,13$, $52,53]$.

While the role of acquired thrombophilia has been accepted as an etiological factor for RPL, the contribution of specific inherited thrombophilic gene polymorphisms is controversial. About $52 \%$ of pregnant women with severe obstetric complications (severe preeclampsia, IUGR, PANPP, stillbirth) had at least one of the three main thrombophilic mutations (FVL G1691A gene mutation, prothrombin G20210A gene mutation, and MTHFR C677T gene mutation) [55]. Among women with congenital thrombophilia (FVL G1691A gene mutation, protein C deficiency, protein $S$ deficiency, AT deficiency, combined defects), fetal loss was found in $29.4 \%$ of cases [56].

A group of thrombogenic gene mutations, especially different combinations thereof: FVL G1691A, factor V H1299R (R2), factor II prothrombin G20210A, factor XIII V34L, $\beta$-fibrinogen G-455A, PAI-1 4G/5G, glycopropein IIIa L33P, MTHFR C677T and MTHFR A1298C can identify people at risk of RPL. Individual examination revealed that 3 of these mutations (PAI-1 4G / 5G, p = 0.009; factor XIII V34L, $\mathrm{p}$ $<0.0001$ and homozygous MTHFR C667T, $\mathrm{p}=0.0001$ ) correlated statistically significantly with RPL, compared to the control group. The other 6 mutant genes, cumulatively, also correlated statistically significantly with RPL $(p<0.0001)$ [57].

Several studies (retrospective, prospective, case-control, cohort, cross-sectional, clinical) and contemporary metaanalyses found no association or found a very weak association with an absolutely very low risk between different forms of congenital thrombophilia or their combinations and RPL $[1,7,11,12,14]$. The most recent and well-designed systematic review and meta-analysis of the association of congenital thrombophilia and placenta-mediated pregnancy complications revealed an absolutely increased, but small, and statistically significant risk of late RPL in women with the FVL G1691A gene mutation [14].

A growing body of evidence (meta-analyses, cross-sectional studies, case-control studies) suggests a significant association of late pregnancy loss and unexplained early RPL with various types of congenital thrombophilia (FVL G1691A gene mutation, prothrombin G20210A gene mutation, MTHFR C677T gene mutation, MTHFR A1298C gene mutation, $\beta$-fibrinogen $\mathrm{G}-455 \mathrm{~A}$ gene mutation, protein $\mathrm{C}$ deficiency, protein $S$ deficiency). Robust evidence for such an association shows the FVL G1691A gene mutation, the prothrombin G20210A gene mutation and hyperhomocysteinemia $[1,3,7,9,11,12,58-61]$.

In three systematic analyses and meta-analyses, which included case-control studies, prospective cohort studies, cross-sectional and randomized controlled trials, only the heterozygous FVL G1691A gene mutation was associated with early and late RPL and late non-recurrent fetal loss, the heterozygous prothrombin G20210A gene mutation - with early RPL and late non-recurrent fetal loss, and protein S deficiency - with RPL and late non-recurrent fetal loss. Protein $\mathrm{C}$ deficiency and AT deficiency have not been associated with fetal loss [13, 62, 63].

A systematic literature review with 16 case-control studies for analysis of FVL G1691A gene mutation and 7 casecontrol studies for analysis of prothrombin G20210A gene mutation, which defined RPL as two or more pregnancy losses in the first or second trimester of pregnancy, found a double risk of RPL in women with congenital thrombophilia compared to women without thrombophilia. The authors concluded that hereditary thrombophilia may be an unrecognized cause of RPL [61].

A meta-analysis, which included 31 population-based studies, showed that the magnitude of the correlation between congenital thrombophilia and fetal loss varies depending on the time of fetal loss and the type of thrombophilia. Only some forms of congenital thrombophilia are associated with fetal loss. In the first trimester, RPL was associated with the FVL G1691A gene mutation and prothrombin G20210A gene mutation, and late non-recurrent fetal loss was associated with the FVL G1691A gene mutation, the prothrombin G20210A gene mutation, and protein S deficiency. The FVL G1691A gene mutation was more strongly associated with late pregnancy loss than with early pregnancy loss, and in the case of exclusion of other potential causes of fetal loss. The MTHFR C677T gene mutation, protein C deficiency, and AT deficiency have not been significantly associated with fetal loss [62].

A large prospective case-control study (European Prospective Cohort on Thrombophilia - EPCOT) enrolled 1384 women (843 women with thrombophilia and 541 control women). The frequency of miscarriage (fetal loss before 28 weeks of gestation) and stillbirth (fetal loss after 28 weeks of gestation) was assessed together and separately. The study found a statistically significant association of pregnancy loss in pregnant women with congenital thrombophilia, and stillbirth especially in women with combined defects or AT deficiency. Women with congenital thrombophilia (AT deficiency, protein $\mathrm{C}$ deficiency and protein S deficiency or FVL G1691A gene mutation) have a 3.6-fold higher risk of stillbirth and a 1.3-fold higher risk of miscarriage. Women with combined birth defects had the highest risk for stillbirth (14.3), compared with 5.2 for AT deficiency, 2.3 for protein C deficiency, 3.3 for protein S deficiency and 2.0 for the FVL G1691A gene mutation. The results obtained have important implications for treatment and provide a justification for clinical trials of thromboprophylaxis in women with RPL [2, 56, 64].

In general, the data described above suggest a clear relationship between congenital thrombophilia and negative pregnancy outcomes $[65,66]$. However, heterogeneous small research groups, differences in study design, ethnicity, and geographical location may impact the results, limiting the statistical power of the research, the validity of the conclusions and the practical importance of the phenomena stud- 
ied $[10,65]$. For these reasons, some scientists believe that the evidence for the association of congenital thrombophilia with pregnancy loss should be interpreted with caution, as it is still weak and unconvincing, lacking the systematic integration of classification and co-factors [10, 65, 67, 68].

Women with combined thrombophilia, especially with congenital thrombophilia associated with acquired thrombophilia or two and more congenital thrombophilias, have the highest risk of early and late RPL $[1,7,13,56,61,69]$. A meta-analysis and some case-control studies confirmed that the FVL G1691A gene mutation is a weak, but well-established risk factor for unexplained RPL (two or more consecutive events) and for one or more late fetal losses with a risk increase of 2-7 times. A significantly increased risk (2.5-fold for early RPL and 2.3-fold for non-recurrent late fetal loss) was also determined in pregnant women with prothrombin G20210A gene mutation. A twofold increase in the risk of fetal loss among pregnant women with AT deficiency, protein C deficiency, protein S deficiency, and FVL G1691A gene mutation has been reported in several studies [70].

A large population study evaluating 32.683 non-thrombotic primigravida women revealed an association of the heterozygous FVL G1691A gene mutation and prothrombin G20210A gene mutation with a significant increased risk of miscarriage (3.2 and 2.4, respectively). The relationship was significant only in the group of women who had a miscarriage from the 10th week of gestation (3.5 and 2.6, respectively) $[51,70,71]$.

In recent years, the association of RPL with hypofibrinolytic gene polymorphisms (coagulation factor XIII V34L, PAI-1 4G / 4G, PAI-1 4G / 5G and ACE I / D gene mutations) has become increasingly evident $[43,72,73]$. Homozygous PAI-1 4G / 5G and MTHFR C677T gene mutations in women with RPL and the FVL G1691A, factor V H1299R, heterozygous ACE I / D gene mutations in both parents play a crucial role in RPL and should be considered risk factors. In addition, RPL is determined by thrombophilic gene mutations in both parents (not just maternal gene mutations) [74]. However, a meta-analysis evaluating 11 studies with a total of 1545 women with RPL and 960 normal women, did not identify any associations of PAI-1 4G/5G gene mutation with idiopathic RPL, including more than two or three recurrent miscarriages, in Caucasian and non-Caucasian population, but a high heterogeneity was identified in the included studies [69].

Therefore, in most cases, the evolution of pregnancy in pregnant women with congenital thrombophilia is devoid of events. However, there is an argument that women with any type of thromboembolic defect, especially with a combination of defects, have a higher prevalence of complications in pregnancy [37]. Although the results of published studies are contradictory, most comments suggest an association of RPL with FVL G1691A gene mutation and prothrombin G20210A gene mutation. These results vary in different populations, reflecting an interaction of several genetic and acquired risk factors $[3,51]$.

Although solid randomized data on this subject are missing, the analysis of existing results revealed that there is no relationship between congenital thrombophilia and RPL, or hereditary thrombophilia has a low impact on placentamediated complications, probably explained by the study design differences, the relatively low prevalence of some forms of congenital thrombophilia and the small number of study participants, differences in inclusion and exclusion criteria, the inclusion of heterogeneous patient populations in different studies and the application of various definitions of complications that did not allow the researchers to identify a significant increase in the risk of RPL. The associations obtained in some studies may be caused by the high frequency of these mutations in the population, which does not reflect the causal relationship [6].

Preeclampsia is an increasingly common, but poorly understood, multisystemic inflammatory syndrome of pregnancy, associated with abnormal placental development and homeostasis disorders leading to inadequate maternal-fetal circulation, caused by endothelial dysfunction, vasoconstriction, placental ischemia, hypercoagulation with uteroplacental thrombosis $[1,11,75]$.

Congenital thrombophilia may partially contribute to the development and severity of preeclampsia due to disorders of the hemostatic system: hypercoagulability with vasculopathy and secondary thrombosis disrupt the perfusion of the intervillous space, contributing to the development of placental infarction, IUGR, PANPP, premature birth and intrauterine fetal death $[2,13,56,76]$.

Some case-control studies and prospective cohort studies (unicenter and multicenter) found the association of total and severe preeclampsia with thrombophilia (congenital and / or acquired). Thrombophilia exacerbates perinatal outcomes in pregnant women with severe preeclampsia: the risk of premature birth (before 32 weeks of gestation), IUGR, and perinatal mortality are significantly higher [3, 77-79].

Data cumulated from several studies and meta-analyses found a significant association of FVL G1691A gene mutation with total preeclampsia (twofold increased risk) and severe preeclampsia (threefold increased risk), prothrombin G20210A gene mutation with total, severe and mild preeclampsia [3, 6, 77, 80-83]. Preeclampsia (total and severe) is the only complication in which a significant and consistent association with homozygosity of MTHFR C677T gene mutation has been found. The presence of a very modest association with mild preeclampsia cannot be excluded, which is very difficult to detect even in significant groups of women $[13,20,65,77,84]$.

A systematic literature review, published in 2002, found an increased risk of preeclampsia / eclampsia in pregnant women with heterozygous FVL G1691A gene mutation, heterozygous prothrombin G20210A gene mutation, homozygous MTHFR C677T gene mutation, protein C deficiency, protein $\mathrm{S}$ deficiency or activated protein $\mathrm{C}$ resistance. Unfortunately, the analysis included many small sample studies, which reduced the overall statistical power and no definitive conclusions could be drawn $[51,85]$.

Two recent extensive meta-analyses, published in 2014 (111 studies with 15094 cases and 21633 controls) and in 2015 (54 studies with 7398 cases and 11222 controls), thus 
guaranteeing the statistical power of meta-analyses and obtaining more accurate estimates, revealed an association of MTHFR C677T gene polymorphism with an increased risk of preeclampsia, especially among Asian and Caucasian population $[21,51]$.

No relationship was found between the deficiency of natural anticoagulants (protein S, protein $\mathrm{C}$ and $\mathrm{AT}$ ) with preeclampsia, as the number of subjects with such defects was too small to allow definitive conclusions to be drawn [3, $65,77]$, although some studies revealed a significant association between protein C and preeclampsia [79].

However, a meta-analysis, published in 2003, and the most recent and well-designed systematic review and metaanalysis of prospective cohort studies did not observe any consistent and significant effect of the FVL G1691A gene mutation and prothrombin G20210A gene mutation on the risk of preeclampsia, although a modest effect cannot be ruled out $[14,65,86]$. While studies published in 1996-2000 demonstrated a significant association of the FVL G1691A gene mutation with preeclampsia, studies published in 20012002 did not provide such convincing evidence, except for a small increased risk attributed to the FVL G1691A gene mutation for preeclampsia. At the same time, it must be acknowledged that the meta-analysis cannot exclude the possibility of associating the FVL G1691A gene mutation with severe forms of preeclampsia [86].

Therefore, the association of preeclampsia with congenital thrombophilia is much more controversial. Currently, there is insufficient evidence to demonstrate the association of any form of congenital thrombophilia with preeclampsia. Several recent studies and meta-analyses suggest that there is an association between congenital thrombophilia and the development of severe preeclampsia but not mild one. Severe preeclampsia is mainly associated with the FVL G1691A gene mutation, hyperhomocysteinemia, protein $S$ and protein C deficiency, AT-III deficiency, prothrombin G20210A gene mutation, and MTHFR C677T gene mutation $[1,11]$.

Intrauterine growth restriction is most commonly defined as a fetal weight, estimated at the ultrasound examination, for gestational age less than the 10th percentile, and severe IUGR - an assessed fetal weight less than the 5th percentile $[10,11]$.

The association between congenital thrombophilia and IUGR or newborns small for gestational age $(<2500 \mathrm{~g})$ has not been consistently demonstrated in various studies and this relationship is highly controversial. The results vary from study to study, depending on the size of the study sample and the selection criteria used. However, a definitively determined causal relationship was not found, although an association was confirmed in women with severe IUGR, but not in cases with mild IUGR $[1,11,12,87,88]$.

Some systematic literature analyses and meta-analyses noticed a significant association between the presence in mothers of the homozygous FVL G1691A gene mutation or the heterozygous prothrombin G20210A gene mutation with IUGR $[8,13,17,52,90]$. None of other congenital prothrombotic defects have been associated with IUGR $[3,13]$.

A systematic literature review, published in 2002, found an increased risk of IUGR in pregnant women with heterozygous prothrombin G20210A gene mutation, homozygous MTHFR C677T gene mutation, protein S deficiency or anticardiolipin antibody IgG isotype. However, these analyses are not definitive due to the small sample size included in the studies and the large confidence interval, which reduced the impact of the findings $[51,85]$. A prospective cohort study, published in 2015, confirmed that the presence of the homozygous MTHFR C677T gene mutation could increase the risk of developing IUGR [89].

Four studies - a family-based case-control study (published in 2002) [88], a large prospective cohort study (published in 2007) [91], a genetic study and a meta-analysis of prospective cohort studies (published in 2008) [81], a metaanalysis of case-control studies and prospective cohort studies (published in 2009) [92] - contradict this association and did not reveal an increased risk of IUGR in pregnant women with polymorphisms of congenital thrombophilia.

Premature abruption of the normally positioned placenta. In general, there is insufficient evidence to establish a link between congenital thrombophilia and PANPP, although a potential association with thrombophilia is suggested by a number of studies. The absence of a consistent association, demonstrated between any type of congenital thrombophilia and PANPP, is possibly caused by the low incidence of these events $[1,11]$.

A systematic literature review, published in 2002, found an increased risk of PANPP in pregnant women with homozygous and heterozygous FVL G1691A gene mutation, heterozygous prothrombin G20210A gene mutation, homocysteinemia and activated protein $\mathrm{C}$ resistance [85].

Some recent systematic literature reviews found a significant risk of PANPP in pregnant women with heterozygous prothrombin G20210A gene mutation, followed by heterozygous FVL G1691A gene mutation and hyperhomocysteinemia $[3,6,13,51]$. A cohort study, published in 2015, confirmed that the presence of FVL G1691A gene mutation could increase the risk of PANPP [89].

However, these results were questioned in a meta-analysis of case-control studies, published in 2008, and a metaanalysis of prospective cohort studies, published in 2010, which did not find any significant risk of premature abruption of the normally positioned placenta in women with FVL G1691A gene mutation or G20210A prothrombin gene mutation or MTHFR C677T gene mutation, except for some evidence of possible genetic causes [3, 14, 27].

Therefore, there is a small amount of robust evidence associated with negative pregnancy outcomes and congenital thrombophilia. The FVL G1691A gene mutation is associated with RPL and late pregnancy loss, prothrombin G20210A gene mutation is associated with RPL and late pregnancy loss, protein S deficiency is associated with late pregnancy loss. There is insufficient evidence to suggest an association of other forms of congenital thrombophilia with pregnancy complications. Due to the poor design quality of case-control studies and prospective cohort studies, there was often an increased relative risk of these complications, especially early RPL, fetal loss and preeclampsia, in women with con- 
genital thrombophilia. However, the magnitude of the association and the absolute risk remain very modest, suggesting that congenital thrombophilia is more of a contributing factor than a primary cause. The existence of a thrombophilic genetic defect does not exclude other risk factors, especially considering the multicausal etiology of obstetric complications in pregnancy. The literature analysis according to the severity of adverse pregnancy outcomes illustrates that the risk associated with congenital thrombophilia increases significantly in pregnant women with severe obstetric complications (severe preeclampsia, eclampsia, PANPP, IUGR less than the 5 th percentile or unexplained stillbirth) $[3,12,89]$.

\section{Conclusions}

1. Prospective cross-sectional, case-control and cohort studies have shown that hereditary thrombophilia is more prevalent in the group of women with recurrent pregnancy loss, late miscarriage, early-onset preeclampsia, premature abruption of the normally positioned placenta, and intrauterine growth restriction.

2. There is convincing evidence that deficiency of natural anticoagulants (antithrombin, protein C, protein S) is a risk factor for late fetal loss. The factor V Leiden G1691A gene mutation and prothrombin G20210A gene mutation are associated with a double risk of recurrent early and unexplained miscarriage as well as non-recurrent late fetal loss. The association of congenital thrombophilia with preeclampsia is limited to the factor V Leiden G1691A gene mutation and more severe cases of preeclampsia. There is insufficient evidence to suggest an association of other forms of congenital thrombophilia with adverse pregnancy outcomes.

3. The risk associated with congenital thrombophilia increases significantly in pregnant women with two or more forms of hereditary thrombophilia and in pregnant women with severe placenta-mediated pregnancy complications (severe preeclampsia, eclampsia, premature abruption of normally positioned placenta, intrauterine growth restriction with weight below the 5th percentile or unexplained stillbirth).

4. Due to the poor design quality of case-control studies and prospective cohort studies, there has often been an increased relative risk of pregnancy complications associated with congenital thrombophilia, especially early RPL, fetal loss and preeclampsia, but the extent of the association and the absolute risk remain very modest. Prospective, multicenter studies are needed on large samples of patients, which would validate the role of thrombophilia in the development of obstetric diseases.

\section{References}

1. Pritchard AM, Hendrix PW, Paidas MJ. Hereditary thrombophilia and recurrent pregnancy loss. Clin Obstet Gynecol. 2016;59(3):487-497. doi: 10.1097/GRF.0000000000000226.

2. Leaf RK, Connors JM. The role of anticoagulants in the prevention of pregnancy complications. Clin Appl Thromb Hemost. 2017;23(2):116-123. doi: 10.1177/1076029615615972.

3. Liatsikos S, Tsikouras P, Manav B, et al. Inherited thrombophilia and reproductive disorders. J Turk Ger Gynecol Assoc. 2016;17(1):45-50. doi: 10.5152/jtgga.2016.15212.
4. Dobbenga-Rhodes Y. Shedding light on inherited thrombophilias: the impact on pregnancy. J Perinat Neonatal Nurs. 2016.;30(1):36-44. doi: 10.1097/JPN.0000000000000146.

5. Alijotas-Reig J, Ferrer-Oliveras R, Esteve-Valverde E, et al. Inherited thrombophilia in women with poor aPL-related obstetric history: prevalence and outcomes. Survey of 208 cases from the European Registry on Obstetric Antiphospholipid Syndrome cohort. Am J Reprod Immunol. 2016;76(2):164-171. doi: 10.1111/aji.12534.

6. Robertson L, Wu O, Greer I. Thrombophilia and adverse pregnancy outcome. Curr Opin Obstet Gynecol. 2004;16(6):453-458. doi: 10.1097/00001703-200412000-00003

7. Tranquilli AL, editor. Thrombophilia. InTech; 2011. 226 p. doi: $10.5772 / 1329$.

8. Davenport WB, Kutteh WH. Inherited thrombophilias and adverse pregnancy outcomes: a review of screening patterns and recommendations. Obstet Gynecol Clin North Am. 2014;41(1):133-144. doi: 10.1016/j. ogc.2013.10.005.

9. Kozma K, Jurca C, Bembea M. Factorii genetici ai trombofiliilor ereditare şi implicarea lor în avortul spontan [The genetic factors of hereditary thrombophilia and their implication in spontaneous abortion]. Rom J Med Pract. 2015;10(2):94-101. Romanian.

10. Duffett L, Rodger M. LMWH to prevent placenta-mediated pregnancy complications: an update. Br J Haematol. 2015;168(5):619-638. doi: 10.1111/bjh.13209.

11. Louis-Jacques A, Maggio L, Romero S. Prenatal screening for thrombophilias: indications and controversies, an update. Clin Lab Med. 2016;36(2):421-434. doi: 10.1016/j.cll.2016.01.016.

12. Simcox L, Ormesher L, Tower C, et al. Thrombophilia and Pregnancy Complications. Int J Mol Sci. 2015;16(12):28418-28428. doi: 10.3390/ ijms 161226104.

13. Robertson L, Wu O, Langhorne P, et al. Thrombophilia in pregnancy: a systematic review. Br J Haematol. 2006;132(2):171-196. doi: 10.1111/j.13652141.2005.05847.x.

14. Rodger M, Betancourt M, Clark P, et al. The association of factor V leiden and prothrombin gene mutation and placenta-mediated pregnancy complications: a systematic review and meta-analysis of prospective cohort studies. PLoS Med. 2010;7(6):e1000292. doi: 10.1371/journal. pmed.1000292.

15. Hemsworth EM, O'Reilly AM, Allen VM, et al. Association between factor v leiden mutation, small for gestational age, and preterm birth: a systematic review and meta-analysis. J Obstet Gynaecol Can. 2016;38(10):897-908. doi: 10.1016/j.jogc.2016.08.001.

16. Ren A, Wang J. Methylenetetrahydrofolate reductase C677T polymorphism and the risk of unexplained recurrent pregnancy loss: a meta-analysis. Fertil Steril. 2006;86(6):1716-1722. doi: 10.1016/j.fertnstert.2006.05.052.

17. Farahmand K, Totonchi M, Hashemi M, et al. Thrombophilic genes alterations as risk factor for recurrent pregnancy loss. J Matern Fetal Neonatal Med. 2016;29(8):1269-1273. doi: 10.3109/14767058.2015.1044431.

18. Cao Y, Xu J, Zhang Z, et al. Association study between methylenetetrahydrofolate reductase polymorphisms and unexplained recurrent pregnancy loss: a meta-analysis. Gene. 2013;514(2):105-111. doi: 10.1016/j. gene.2012.10.091

19. Wu X, Zhao L, Zhu H, et al. Association between the MTHFR C677T polymorphism and recurrent pregnancy loss: a meta-analysis. Genet Test Mol Biomarkers. 2012;16(7):806-811. doi: 10.1089/gtmb.2011.0318.

20. Yang B, Fan S, Zhi X, et al. Associations of MTHFR gene polymorphisms with hypertension and hypertension in pregnancy: a metaanalysis from 114 studies with 15411 cases and 21970 controls. PLoS One. 2014;9(2):e87497. doi: 10.1371/journal.pone.0087497.

21. Wu X, Yang K, Tang X, et al. Folate metabolism gene polymorphisms MTHFR C677T and A1298C and risk for preeclampsia: a meta-analysis. J Assist Reprod Genet. 2015;32(5):797-805. doi: 10.1007/s10815-0140408-8.

22. Nurk E, Tell G, Refsum H, et al. Associations between maternal methylenetetrahydrofolate reductase polymorphisms and adverse outcomes of pregnancy: the Hordaland Homocysteine Study. Am J Med. 2004;117(1):26-31. doi: 10.1016/j.amjmed.2004.01.019.

23. Aracic N, Roje D, Drmic Hofman I, et al. Low molecular weight heparin treatment and impact of inherited thrombophilia type in pregnancies with previous adverse outcome. J Matern Fetal Neonatal Med. 2015;28(3):306310. doi: $10.3109 / 14767058.2014 .916268$. 
24. Liguori R, Quaranta S, Di Fiore R, et al. A novel polymorphism in the PAI-1 gene promoter enhances gene expression. A novel pro-thrombotic risk factor? Thromb Res. 2014;134(6):1229-1233. doi: 10.1016/j. thromres.2014.09.021.

25. Pecheniuk NM, Morris CP, Walsh TP, et al. The factor V HR2 haplotype: prevalence and association of the A4070G and A6755G polymorphisms. Blood Coagul Fibrinolysis. 2001;12(3):201-206. doi: 10.1097/00001721200104000-00006.

26. Nair R, Khanna A, Singh R, et al. Association of maternal and fetal MTHFR A1298C polymorphism with the risk of pregnancy loss: a study of an Indian population and a meta-analysis. Fertil Steril. 2013;99(5):1311-1318.e4. doi: 10.1016/j.fertnstert.2012.12.027.

27. Zdoukopoulos N, Zintzaras E. Genetic risk factors for placental abruption: a HuGE review and meta-analysis. Epidemiology. 2008;19(2):309-323. doi: 10.1097/EDE.0b013e3181635694.

28. Jeddi-Tehrani M, Torabi R, Zarnani A, et al. Analysis of plasminogen activator inhibitor-1, integrin beta3, beta fibrinogen, and methylenetetrahydrofolate reductase polymorphisms in Iranian women with recurrent pregnancy loss. Am J Reprod Immunol. 2011;66(2):149-156. doi: 10.1111/j.1600-0897.2010.00974.x.

29. Yousefian E, Kardi M, Allahveisi A. Methylenetetrahydrofolate reductase C677T and A1298C polymorphism in Iranian women with idiopathic recurrent pregnancy losses. Iran Red Crescent Med J. 2014;16(7):e16763. doi: 10.5812/ircmj.16763.

30. Poursadegh Zonouzi A, Chaparzadeh N, Asghari Estiar M, et al. Methylenetetrahydrofolate reductase C677T and A1298C mutations in women with recurrent spontaneous abortions in the Northwest of Iran. ISRN Obstet Gynecol. 2012;2012:945486. doi: 10.5402/2012/945486.

31. Li WX, Dai SX, Zheng JJ, et al. Homocysteine metabolism gene polymorphisms (MTHFR C677T, MTHFR A1298C, MTR A2756G and MTRR A66G) jointly elevate the risk of folate deficiency. Nutrients. 2015;7(8):6670-6687. doi: 10.3390/nu7085303.

32. Li W, Cheng F, Zhang A, et al. Folate deficiency and gene polymorphisms of MTHFR, MTR and MTRR elevate the hyperhomocysteinemia risk. Clin Lab. 2017;63(3):523-533. doi: 10.7754/Clin.Lab.2016.160917.

33. Said JM, Higgins JR, Moses EK, et al. Inherited thrombophilia polymorphisms and pregnancy outcomes in nulliparous women. Obstet Gynecol. 2010;115(1):5-13. doi: 10.1097/AOG.0b013e3181c68907.

34. Franco R, Maffei F, Lourenço D, et al. The frequency of 844 ins 68 mutation in the cystathionine beta-synthase gene is not increased in patients with venous thrombosis. Haematologica. 1998;83(11):1006-1008.

35. Popp R, Crișan T, Rotar I, et al. Cystathionine $\beta$-synthase 844 ins 68 genetic polymorphism in spontaneous abortion susceptibility. Appl Med Inform. 2011;29(4):34-40.

36. Yakub M, Moti N, Parveen S, et al. Polymorphisms in MTHFR, MS and CBS genes and homocysteine levels in a Pakistani population. PLoS One. 2012;7(3):e33222. doi: 10.1371/journal.pone.0033222.

37. Barbosa PR, Stabler SP, Machado AL, et al. Association between decreased vitamin levels and MTHFR, MTR and MTRR gene polymorphisms as determinants for elevated total homocysteine concentrations in pregnant women. Eur J Clin Nutr. 2008;62(8):1010-1021. doi: 10.1038/ sj.ejcn. 1602810 .

38. Song CS, Song WB, Bao JY, et al. Association between decreased plasma folate levels and MTHFR C677T, and MTRR A66G gene polymorphisms as determinants for elevated total homocysteine concentrations in pregnant women. Hered Genet Curr Res. 2018;7(1):1000193. doi: 10.4172/21611041.1000193

39. Torabi R, Zarei S, Zeraati H, et al. Combination of thrombophilic gene polymorphisms as a cause of increased risk of recurrent pregnancy loss. J Reprod Infertil. 2012;13(2):89-94.

40. $\mathrm{Li} \mathrm{J}, \mathrm{Wu} \mathrm{H}$, Chen Y, et al. Genetic association between FXIII and $\beta$-fibrinogen genes and women with recurrent spontaneous abortion: a meta-analysis. J Assist Reprod Genet. 2015;32(5):817-825. doi: 10.1007/ s10815-015-0471-9.

41. Wang J, Wang C, Chen $\mathrm{N}$, et al. Association between the plasminogen activator inhibitor-1 4G/5G polymorphism and risk of venous thromboembolism: a meta-analysis. Thromb Res. 2014;134(6):1241-1248. doi: 10.1016/j.thromres.2014.09.035.

42. Chen H, Nie S, Lu M. Association between plasminogen activator inhibitor-1 gene polymorphisms and recurrent pregnancy loss: a systematic review and meta-analysis. Am J Reprod Immunol. 2015;73(4):292-300. doi: 10.1111/aji.12321.

43. Li X, Liu Y, Zhang R, et al. Meta-analysis of the association between plasminogen activator inhibitor-1 4G/5G polymorphism and recurrent pregnancy loss. Med Sci Monit. 2015;(21):1051-1056. doi: 10.12659/ MSM.892898.

44. Magdoud K, Herbepin V, Touraine R, et al. Plasminogen activator inhibitor $14 \mathrm{G} / 5 \mathrm{G}$ and $-844 \mathrm{G} / \mathrm{A}$ variants in idiopathic recurrent pregnancy loss. Am J Reprod Immunol. 2013;70(3):246-252. doi: 10.1111/aji.12116.

45. Lino F, Traina E, Barreto J, et al. Thrombophilic mutations and polymorphisms, alone or in combination, and recurrent spontaneous abortion. Clin Appl Thromb Hemost. 2015;21(4):365-372. doi: $10.1177 / 1076029613520465$.

46. Barlik M, Seremak-Mrozikiewicz A, Drews K, et al. Correlation between factor VII and PAI-1 genetic variants and recurrent miscarriage. Ginekol Pol. 2016;87(7):504-509. doi: 10.5603/GP.2016.0034.

47. Salazar Garcia M, Sung N, Mullenix T, et al. Plasminogen activator inhibitor-1 4G/5G polymorphism is associated with reproductive failure: metabolic, hormonal, and immune profiles. Am J Reprod Immunol. 2016;76(1):70-81. doi: 10.1111/aji.12516.

48. Mehdi S, Khosravi A, Pakravesh J, et al. Factor XIII Val34Leu polymorphism and risk of recurrent pregnancy loss in Iranian population: a case-control study. Frontier Biol. 2016;11(6):471-475. doi: 10.1007/ s11515-016-1419-x.

49. Bagheri M, Rad I, Omrani M, et al. The Val34Leu genetic variation in the A subunit of coagulation factor XIII in recurrent spontaneous abortion. Syst Biol Reprod Med. 2011;57(5):261-264. doi: 10.3109/19396368.2011.576308.

50. Huchon C, Deffieux X, Beucher G, et al. Pregnancy loss: French clinical practice guidelines. Eur J Obstet Gynecol Reprod Biol. 2016;201:18-26. doi: 10.1016/j.ejogrb.2016.02.015.

51. Battinelli E, Marshall A, Connors J. The role of thrombophilia in pregnancy. Thrombosis. 2013;2013:516420. doi: 10.1155/2013/516420.

52. de Jong P, Goddijn M, Middeldorp S. Antithrombotic therapy for pregnancy loss. Hum Reprod Update. 2013;19(6):656-673. doi: 10.1093/ humupd/dmt019.

53. Garrido-Gimenez C, Alijotas-Reig J. Recurrent miscarriage: causes, evaluation and management. Postgrad Med J. 2015;91(1073):151-162. doi: 10.1136/postgradmedj-2014-132672.

54. Kaiser J, Branch D. Recurrent pregnancy loss: generally accepted causes and their management. Clin Obstet Gynecol. 2016;59(3):464-473. doi: 10.1097/GRF.0000000000000214

55. Kupferminc M, Eldor A, Steinman N, et al. Increased frequency of genetic thrombophilia in women with complications of pregnancy. N Engl J Med. 1999;340(1):9-13. doi: 10.1056/NEJM199901073400102.

56. Preston FE, Rosendaal FR, Walker ID, et al. Increased fetal loss in women with heritable thrombophilia. Lancet. 1996;348(9032):913-916. doi: 10.1016/s0140-6736(96)04125-6.

57. Goodman CS, Coulam CB, Jeyendran RS, et al. Which thrombophilic gene mutations are risk factors for recurrent pregnancy loss? Am J Reprod Immunol. 2006;56(4):230-236. doi: 10.1111/j.1600-0897.2006.00419.x.

58. Lee GS, Park JC, Rhee JH, et al. Etiologic characteristics and index pregnancy outcomes of recurrent pregnancy losses in Korean women. Obstet Gynecol Sci. 2016;59(5):379-387. doi: 10.5468/ogs.2016.59.5.379.

59. Patil R, Ghosh K, Vora S, et al. Inherited and acquired thrombophilia in Indian women experiencing unexplained recurrent pregnancy loss. Blood Cells Mol Dis. 2015;55(3):200-205. doi: 10.1016/j.bcmd.2015.06.008.

60. Osman OM, Abulata NN. Inherited thrombophilia and early recurrent pregnancy loss among Egyptian women. Open J Obstet Gynecol. 2015;5(5):251-258.

61. Kovalevsky G, Gracia C, Berlin J, et al. Evaluation of the association between hereditary thrombophilias and recurrent pregnancy loss: a meta-analysis. Arch Intern Med. 2004;164(5):558-563. doi: 10.1001/ archinte.164.5.558.

62. Rey E, Kahn S, David M, et al. Thrombophilic disorders and fetal loss: a meta-analysis. Lancet. 2003;361(9361):901-908. doi: 10.1016/S01406736(03)12771-7.

63. Gao H, Tao F. Prothrombin G20210A mutation is associated with recurren pregnancy loss: a systematic review and meta-analysis update. Thromb Res. 2015;135(2):339-346. doi: 10.1016/j.thromres.2014.12.001. 
64. Vossen C, Preston F, Conard J, et al. Hereditary thrombophilia and fetal loss: a prospective follow-up study. J Thromb Haemost. 2004;2(4):592-596. doi: 10.1111/j.1538-7836.2004.00662.x.

65. Greer I, Brenner B, Gris J. Antithrombotic treatment for pregnancy complications: which path for the journey to precision medicine? $\mathrm{Br}$ Haematol. 2014;165(5):585-599. doi: 10.1111/bjh.12813.

66. Silan F, Mosse I, Gonchar A, et al. Comparison of the thrombophilic gene polymorphisms and recurrent pregnancy loss: Results on combined gene effect of FV Leiden, FVR2, FXIII, MTHFR (A1298C and C677T), PAI-1 4G/5G and ACE I/D genes in RPL Women from Minsk/Belarus and Canakkale - Sivas/Turkey. Biomed Genet Genomics. 2016;1(4):87-93. doi: 10.15761/BGG.1000117.

67. Bennett S, Bagot C, Arya R. Pregnancy loss and thrombophilia: the elusive link. Br J Haematol. 2012;157(5):529-542. doi: 10.1111/j.13652141.2012.09112.x.

68. Rodger M, Paidas M. Do thrombophilias cause placenta-mediated pregnancy complications? Semin Thromb Hemost. 2007;33(6):597-603. doi: 10.1055/s-2007-985756.

69. Su MT, Lin SH, Chen YC, et al. Genetic association studies of ACE and PAI-1 genes in women with recurrent pregnancy loss: a systematic review and meta-analysis. Thromb Haemost. 2013;109(1):8-15. doi: 10.1160/ TH12-08-0584.

70. De Stefano V, Rossi E, Za T. Inherited thrombophilia and obstetric complications. Haematol Rep. 2005;1(10):18-21.

71. Lissalde-Lavigne G, Fabbro-Peray P, Cochery-Nouvellon E, et al. Factor V Leiden and prothrombin G20210A polymorphisms as risk factors for miscarriage during a first intended pregnancy: the matched case-control 'NOHA first' study. J Thromb Haemost. 2005;3(10):2178-2184. doi: 10.1111/j.1538-7836.2005.01581.x.

72. Dossenbach-Glaninger A, van Trotsenburg M, Schneider B, et al. ACE I/D polymorphism and recurrent first trimester pregnancy loss: interaction with SERPINE1 4G/5G and F13 Val34Leu polymorphisms. Br J Haematol. 2008;141(2):269-271. doi: 10.1111/j.1365-2141.2008.07058.x.

73. Shakarami F, Akbari M, Zare Karizi S. Association of plasminogen activator inhibitor-1 and angiotensin converting enzyme polymorphisms with recurrent pregnancy loss in Iranian women. Iran J Reprod Med. 2015;13(10):627-632.

74. Ozdemir O, Yenicesu G, Silan F, et al. Recurrent pregnancy loss and its relation to combined parental thrombophilic gene mutations. Genet. Test. Mol. Biomarkers. 2012;16(4):279-286. doi: 10.1089/gtmb.2011.0191.

75. Wilson ML, Goodwin TM, Pan VL, et al. Molecular epidemiology of preeclampsia. Obstet Gynecol Surv. 2003;58(1):39-66. doi: 10.1097/00006254200301000-00022.

76. Giannubilo S, Landi B, Ciavattini A. Preeclampsia: what could happen in a subsequent pregnancy? Obstet Gynecol Surv. 2014;69(12):747-762. doi: 10.1097/OGX.0000000000000126.

77. Mello G, Parretti E, Marozio L, et al. Thrombophilia is significantly associated with severe preeclampsia: results of a large-scale, casecontrolled study. Hypertension. 2005;46(6):1270-1274. doi: 10.1161/01. HYP.0000188979.74172.4d.
78. Kupferminc M, Fait G, Many A, et al. Severe preeclampsia and high frequency of genetic thrombophilic mutations. Obstet Gynecol. 2000;96(1):45-49. doi: 10.1016/s0029-7844(00)00861-9.

79. Saghafi N, Mohammadzadeh Vatanchi A, Tara F, et al. Evaluation of selected thrombotic factors among pregnant women with preeclampsia and normal pregnant women. Iran J Reprod Med. 2014;12(12):793-798.

80. Fong F, Sahemey M, Hamedi G, et al. Maternal genotype and severe preeclampsia: a HuGE review. Am J Epidemiol. 2014;180(4):335-345. doi: 10.1093/aje/kwu151.

81. Dudding T, Heron J, Thakkinstian A, et al. Factor V Leiden is associated with pre-eclampsia but not with fetal growth restriction: a genetic association study and meta-analysis. J Thromb Haemost. 2008;6(11):1868-1875. doi: 10.1111/j.1538-7836.2008.03134.x.

82. Wang X, Bai T, Liu S, et al. Association between thrombophilia gene polymorphisms and preeclampsia: a meta-analysis. PLoS One. 2014;9(6):e100789. doi: 10.1371/journal.pone.0100789.

83. Lin J, August P. Genetic thrombophilias and preeclampsia: a metaanalysis. Obstet Gynecol. 2005;105(1):182-192. doi: 10.1097/01. AOG.0000146250.85561.e9.

84. Niu W, You Y, Qi Y. Strong association of methylenetetrahydrofolate reductase gene C677T polymorphism with hypertension and hypertensionin-pregnancy in Chinese women: a meta-analysis. J Hum Hypertens. 2012;26(4):259-267. doi: 10.1038/jhh.2011.11.

85. Alfirevic Z, Roberts D, Martlew V. How strong is the association between maternal thrombophilia and adverse pregnancy outcome? A systematic review. Eur J Obstet Gynecol Reprod Biol. 2002;101(1):6-14. doi: 10.1016/ s0301-2115(01)00496-1.

86. Kosmas I, Tatsioni A, Ioannidis J. Association of Leiden mutation in factor $\mathrm{V}$ gene with hypertension in pregnancy and pre-eclampsia: a meta-analysis. J Hypertens. 2003;21(7):1221-1228. doi: 10.1097/00004872200307000-00002.

87. McCowan L, Craigie S, Taylor R, et al. Inherited thrombophilias are not increased in «idiopathic» small-for-gestational-age pregnancies. Am J Obstet Gynecol. 2003;188(4):981-985. doi: 10.1067/mob.2003.218.

88. Infante-Rivard C, Rivard G, Yotov W, et al. Absence of association of thrombophilia polymorphisms with intrauterine growth restriction. N Engl J Med. 2002;347(1):19-25. doi: 10.1056/NEJM200207043470105.

89. Livrinova V, Lega M, Dimcheva A, et al. Factor V Leiden, prothrombin and MTHFR Mutation in patients with preeclamsia, intrauterine growth restriction and placental abruption. Open Access Maced J Med Sci. 2015;3(4):590-594. doi: 10.3889/oamjms.2015.099.

90. Coriu L, Copaciu E, Tulbure D, et al. Inherited thrombophilia in pregnant women with intrauterine growth restriction. Maedica (Bucharest). 2014;9(4):351-3

91. Clark P, Walker I, Govan L, et al. The GOAL study: a prospective examination of the impact of factor $\mathrm{V}$ Leiden and $\mathrm{ABO}(\mathrm{H})$ blood groups on haemorrhagic and thrombotic pregnancy outcomes. Br J Haematol. 2008;140(2):236-240. doi: 10.1111/j.1365-2141.2007.06902.x.

92. Facco F, You W, Grobman W. Genetic thrombophilias and intrauterine growth restriction: a meta-analysis. Obstet Gynecol. 2009;113(6):12061216. doi: 10.1097/AOG.0b013e3181a6e96a.

\section{Authors' ORCID iDs and academic degrees}

Valentin Friptu, MD, PhD, Professor of Gynecology and Obstetrics - https://orcid.org/0000-0003-2804-899X

Diana Mitriuc, MD, PhD Applicant - https://orcid.org/0000-0002-5146-8012

Olga Popusoi, MD, PhD, Associate Professor of Gynecology and Obstetrics - https://orcid.org/0000-0002-8676-5092

\section{Authors' contributions}

VF conceptualized the idea and revised the article critically; DM conducted literature review, and wrote the first manuscript; OP revised, added and completed the final text. All the authors approved the final version of the manuscript.

\section{Funding}

This study was supported by Nicolae Testemitanu State University of Medicine and Pharmacy. The review study was the authors' initiative. The authors are independent and take responsibility for the integrity of the data and accuracy of the data analysis.

Ethics approval and consent to participate. No approval was required for this study.

Conflict of Interests. The authors have no conflict of interests to declare. 